\title{
Estudo Prospectivo sobre Tecnologia Assistiva na Educação Escolar para Criança com Deficiência Intelectual/Mental
}

\author{
Prospective Study on Assistive Technology in School Education for \\ Children With Intellectual /Mental Deficiency
}

\author{
Júlia Maciela Oliveira de Tassis Frasson Cardozo ${ }^{1}$ \\ Cristiane Xavier Galhardo ${ }^{1}$ \\ Vivianni Marques Leite dos Santos ${ }^{1}$ \\ ${ }^{1}$ Universidade Federal do Vale do São Francisco, Juazeiro, BA, Brasil
}

\begin{abstract}
Resumo
Tecnologia Assistiva (TA) é o campo do conhecimento, com característica interdisciplinar, que por meio do uso de produtos e serviços assistivos tem como objetivo promover a funcionalidade relacionada à participação $e$ à atividade de pessoas com deficiência, mobilidade reduzida ou incapacidades, visando a sua independência, autonomia, qualidade de vida e inclusão social. É um dos meios pelos quais se pode alcançar a aplicação da Lei Brasileira de Inclusão da Pessoa com Deficiência. Assim, este artigo pretende realizar uma prospecção tecnológica do panorama mundial da proteção de produtos e processos associados à TA, investigando as patentes por país de origem, evolução anual, tipo de instituição e empresas envolvidas no desenvolvimento. Para tanto, foram utilizadas as ferramentas Matriz SWOT e Roadmap Tecnológico. Constatou-se que, apesar de a TA contemplar potencialidades nos mais diferentes ramos da tecnologia, é incipiente o número de empresas e de universidades atuando nesta área, demonstrando a necessidade de fomento na pesquisa, no desenvolvimento $e$ na inovação do setor.
\end{abstract}

Palavras-chave: Déficit Intelectual. Legislação Inclusiva. Tecnologia Inclusiva.

\begin{abstract}
Assistive Technology (AT) is an interdisciplinary field of knowledge that, through the use of assistive products and services, aims to promote the functionality related to the participation and activity of people with disabilities, reduced mobility or disabilities, aiming at their independence, autonomy, quality of life and social inclusion. It is one of the means by which the application of the Brazilian Law for the Inclusion of Persons with Disabilities can be achieved. Thus, the objective is to carry out technological prospection of the world panorama of protection of products and processes associated with AT, investigating the patents by country of origin, annual evolution, type of institution and companies involved in the development. For that, the SWOT Matrix and Technological Roadmap tools were used. Despite the fact that AT has potentialities in the most different branches of technology, the number of companies and universities operating in this area is incipient, demonstrating the need to foster research, development and innovation in the sector.
\end{abstract}

Keywords: Intellectual Deficit. Inclusive Legislation. Inclusive Technology.

Área Tecnológica: Prospecções Nacionais e Internacionais. Tecnologia Assistiva Educacional. 


\section{Introdução}

De acordo com a Lei Brasileira de Inclusão da Pessoa com Deficiência - Estatuto da Pessoa com Deficiência (Lei n. 13.416/2015) - pessoa com deficiência (PcD) é aquela que tem impedimento de longo prazo, de natureza, sensorial, mental, física ou intelectual, que em contato com uma ou mais barreiras, estas podem bloquear sua atividade plena e efetiva na coletividade em igualdade de condições com as demais pessoas.

Segundo levantamento feito pelo Instituto Brasileiro de Geografia e Estatística (IBGE, 2019), quase $24 \%$ da população brasileira têm algum tipo de deficiência (KINSTSCHNER; FERREIRA, 2018). Nesse mesmo sentido, a Pesquisa Nacional de Saúde (PNS, 2013), em parceria com o Ministério da Saúde, por meio de consulta a 64 mil domicílios, declarou quatro tipos de deficiências: auditiva, visual, física e intelectual/mental.

O IBGE (2019) apresentou por meio do Censo Demográfico, 2010, um total de 2.611.536 pessoas com deficiência metal/intelectual. Dessa soma, percebeu-se que as Unidades da Federação, Pernambuco e Bahia têm, respectivamente, 138.677 e 211.402 pessoas com deficiência mental/intelectual, sendo que desse total $0,5 \%$ já nasceram com as limitações. Do total de pessoas com deficiência intelectual, mais da metade, ou seja, 54,8\% têm grau intenso ou muito intenso de limitação e cerca de $30 \%$ frequentam algum serviço de reabilitação em saúde. De acordo com o IBGE, para o censo de 2020 será aplicado um "questionário-amostra" em 10\% dos domicílios particulares permanentes do país, o que equivale a cerca de 7,1 milhões de domicílios brasileiros. Neste questionário será abordado o item "Pessoas com deficiência", que trará resultados mais atuais, apesar da representação (IBGE, 2019).

Frente às dificuldades diárias do público-alvo e de seus familiares, foi instituída a Lei Brasileira de Inclusão da Pessoa com Deficiência (Estatuto da Pessoa com Deficiência), Lei n. 13.146, de 6 de julho de 2015, destinada a promover e a assegurar, em condições de igualdade, o exercício dos direitos e das liberdades fundamentais por pessoa com deficiência, visando a sua inclusão social e cidadania.

O Estatuto da $\mathrm{PcD}$, em seu artigo $4^{\circ}$, caput, afirma que "[...] toda pessoa com deficiência tem direito à igualdade de oportunidades com as demais pessoas e não sofrerá nenhuma espécie de discriminação". Porém para fazer jus a esses direitos, são necessárias algumas diretrizes, dentre elas a atuação permanente, integrada e articulada de políticas públicas que possibilitem a plena participação social da pessoa com deficiência (BRASIL, 2015).

Logo, é dever do Estado, da sociedade e da família assegurar à pessoa com deficiência, com prioridade, a efetivação dos direitos referentes à educação, profissionalização, ao trabalho, à habilitação e à reabilitação, à acessibilidade, à informação, à comunicação, aos avanços científicos e tecnológicos, à dignidade, ao respeito, à liberdade, à convivência familiar e comunitária, entre outros decorrentes da Constituição Federal/1988, da Convenção sobre os Direitos das Pessoas com Deficiência e seu Protocolo Facultativo e das leis e de outras normas que garantam seu bem-estar pessoal, social e econômico (BRASIL, 2015).

O emprego de recursos tecnológicos como meio para a melhoria da acessibilidade da pessoa com deficiência intelectual é uma ação que busca minimizar, neutralizar ou eliminar as barreiras que a impedem no seu processo de aprendizagem e interação no convívio social. Consequen- 
temente, um progresso gradativo por meio de acompanhamento e interação de instituição de ensino, família e sociedade.

O termo Tecnologia Assistiva (TA) ainda é novo, sendo utilizado para identificar todo o arsenal de recursos e serviços que contribuem para proporcionar ou ampliar habilidades funcionais de $\mathrm{PcD}$ e, consequentemente, promover vida independente $e$ inclusão. $\mathrm{O}$ objetivo da TA é proporcionar à $\mathrm{PCD}$ maior independência, qualidade de vida e inclusão social, por meio da ampliação de sua comunicação, mobilidade, controle de seu ambiente, habilidades de seu aprendizado e trabalho (BERSCH, 2017).

Nessa circunstância, o desenvolvimento de TA para a promoção da inclusão social da PcD e o favorecimento da entrada destes produtos no mercado nacional, gera preocupações quanto à proteção dessas tecnologias. O Instituto Nacional da Propriedade Industrial (INPI), desde 1970 é responsável em disseminar, aperfeiçoar e gerir o sistema brasileiro de garantia e concessão do direito de propriedade intelectual para a indústria, com os serviços de registros de: indicações geográficas, marcas, desenhos industriais, programas de computador e topografias de circuitos, as averbações de contratos de franquia, as concessões de patentes e transferência de tecnologia.

O avanço do estudo para prospectar TA surgiu da necessidade de aprofundar o conhecimento de alguns aspectos, tanto da demanda quanto da oferta, para o atendimento de necessidades especiais dos diversos segmentos da população, sobre os quais se carece, ainda, de informações sistematizadas e abrangentes (CGEE, 2014).

A realização de busca em banco de dados, por registros de patentes anteriores à invenção, não tem apenas a função de apoio na elaboração do documento de patente, entretanto pode ser utilizado para as mais diversas atividades do meio acadêmico, como a elaboração de prospecção tecnológica, mapeamento de novas tecnologias aplicadas em determinadas áreas de desenvolvimento, percepção de tendências, através do estado da técnica e histórico do invento, análise de originalidade das propostas de trabalhos científicos e de pesquisa, busca de novos temas para trabalhos e nichos de mercado com interesse na tecnologia desenvolvida (COELHO, 2003).

Portanto, visualizar o comportamento do mercado mundial frente à tecnologia e aos principais envolvidos permite o direcionamento de futuros projetos nessa área. Cardoso, Bomtempo e Borschiver (2017) destacam que a análise prospectiva de tecnologias vem sendo cada vez mais utilizada para descrever os fluxos e o desdobramento de novas tecnologias e de tomada de decisão, pela análise dos padrões de patentes.

Assim, por meio do estudo prospectivo, este artigo visa a identificar as tecnologias que já estão no mercado e estabelecer características de novos produtos em tecnologia assistiva na educação escolar, sob a perspectiva da Matriz SWOT e com auxílio de Roadmap Tecnológico.

\section{Metodologia}

O presente estudo foi constituído de três etapas para alcançar o melhor resultado possível para a prospecção. A etapa inicial, conhecida também como Pré-Prospectiva, deu-se por busca casual sobre o tema discutido para alcançar uma visão geral do estado da arte, com o uso da Technology Roadmap e da Matriz SWOT para a compreensão das informações obtidas a partir do estudo, e assim atingir o objetivo do trabalho. A Figura 1 mostra as etapas da pesquisa realizada. 
Figura 1 - Metodologia para a elaboração da Matriz SWOT e Roadmap tecnológico

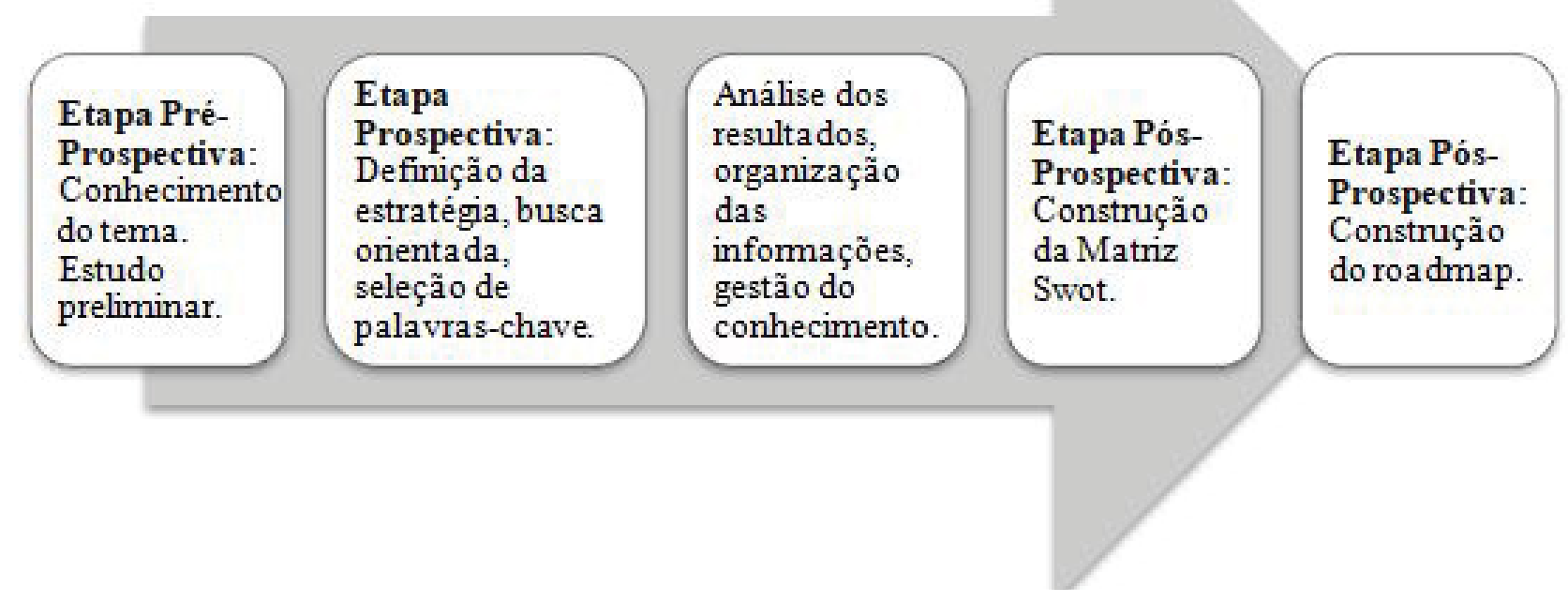

Fonte: Adaptada de Borschiver e Lemos (2016)

Na segunda etapa, como forma de busca orientada e gestão do conhecimento, foi indispensável realizar um levantamento de palavras-chave. A partir das palavras escolhidas realizou-se uma patentometria cuja busca foi realizada em três bases, sendo duas nacionais, Instituto Nacional da Propriedade Industrial e Portal Periódicos CAPES/ MEC, e uma internacional, Orbit Questel, que abrange bases de patentes de mais de 96 países.

Para a realização de busca, foram utilizadas em todas as bases, as palavras-chaves da seguinte forma: "defic* intelectual", "deficiência intelectual", "deficiência and intelectual", "deficiência intelectual/ mental", "portador de necessidade especial", "tecnologia assistiva", "tecnologia assistiva educacional", "tecnologia inclusiva", "tecnologia inclusiva educacional". Para facilitar a filtragem, foram utilizados o caractere de truncagem e o termo "and" para melhorar os resultados das pesquisas.

Com intuito de avaliar o panorama mundial da proteção das inovações relacionadas à TA, foram investigados os documentos de patentes depositados por país de origem, a evolução anual de depósito, o tipo de instituição que protegem, bem como as principais empresas envolvidas no desenvolvimento dessa tecnologia no mundo.

Ainda na segunda etapa (prospectiva), dentro do periódico Capes, por busca avançada, por título, com data da publicação entre os anos 2010 a 2018, buscou-se analisar artigos científicos que demonstrassem algum grau de desenvolvimento da tecnologia, para as mesmas palavras-chaves: "defic* intelectual", "deficiência intelectual", "deficiência and intelectual", "deficiência intelectual/ mental", "portador de necessidade especial", "tecnologia assistiva", "tecnologia assistiva educacional", "tecnologia inclusiva", "tecnologia inclusiva educacional" (BORSCHIVER; LEMOS, 2016).

A coleta dos dados foi feita entre os dias 29 de novembro a 17 de dezembro de 2018, os quais foram organizados e tabulados com o auxílio do sistema Orbit e das ferramentas das bases de patentes, bem como através da utilização do MS Excel 2013 e do Word. 
Dessa forma, caracteriza-se a pesquisa por ser exploratória em base de dados e natureza ex-post fact, uma vez que os dados foram extraídos após a ocorrência dos fatos com a utilização de fenômenos produzidos por terceiros, valendo-se de uma amostra intencional com uso de variáveis descritivas enumerando as diversas qualidades do objeto de estudo e mensurando ainda o quantitativo de patentes e documentos associados a este elemento de pesquisa (TATUM et al., 2018).

Na terceira etapa, conhecida também como etapa Pós-Prospectiva, as informações analisadas foram mapeadas, destacando-se visualmente os aspectos mais relevantes do estudo, assim como a inter-relação entre as informações. O modelo adotado para esta pesquisa é o genérico proposto por Phaal, que consiste em representação gráfica baseada no tempo e compreende um número de camadas e subcamadas que, tipicamente, incluem perspectivas comerciais $e$ tecnológicas (BORSCHIVER; LEMOS, 2016).

\section{Resultados e Discussão}

Quanto à realização da análise patentária, pôde-se localizar nacionalmente na base do INPI, em pesquisa avançada, com o uso das palavras-chave "tecnologia assistiva", 358 processos, sendo que com apenas a palavra-chave "assistiva", foram encontrados três processos, um com data de depósito de 25 de abril de 2017, publicado em 21 de novembro de 2018.

As buscas acima tratam: de um equipamento de TA para auxiliar pessoas com mobilidade reduzida na mão para efetuar escovação dental (BR 102017008477 9); a segunda, depositada em 15 de dezembro de 2014 e publicada em 19 de julho de 2016, trata-se de uma TA para acessibilidade de deficientes físicos ao computador (BR 102014031348 6); e a terceira, depositada em 10 de novembros de 2010 e publicada em 26 de dezembro de 2013, refere-se a uma prótese com dispositivo intrabucal de acionamento de sensores de reprodução de som da fala para a comunicação assistiva (PI 1004775-1).

Com as palavras-chave "assistiva educacional", em pesquisa avançada, busca em título, foram localizados 60 processos. Desses processos, apenas um está relacionado à (cognição): BR 1120130241586 A2; com patente depositada em 22 de março de 2012 e publicada em 6 de dezembro de 2016; com prioridade unionista para os Estados Unidos em 22 de março de 2011 a 21 de março de 12, sendo arquivada em 20 de junho de 2017. Trata-se de tecnologia referente à análise de elementos de resultado educacional cognitivo, relacionada ao relatório interativo, contudo não direcionado ao público com déficit de atenção para acompanhamento em escola/família/assistência médico.

Posteriormente, foi realizada investigação com as palavras-chave "tecnologia inclusiva", e a pesquisa apresentou 362 resultados englobando vários ramos tecnológicos; para "tecnologia inclusiva educacional" foram localizados 415 resultados; "Inclusiva educacional" foram encontradas 58 patentes inclusivas na área da educação, porém tecnologias com abrangência para várias tipos de deficiências e não foi encontrada tecnologia protegida para o público-alvo, com aplicação na seara da educação escolar.

Quanto à prospecção feita a partir de busca na base do Portal de Periódicos CAPES/MEC, foi realizada uma pesquisa avançada, para artigos científicos, com a utilização do referencial data, entre os anos de 2010 a 2018, caractere de truncagem e a palavra and, para as palavras- 
-chave "defic* intelectual", "deficiência and intelectual", e foram encontrados 4.134 e 1.169 artigos, respectivamente, conforme pode ser verificado na Tabela 1.

Com a análise dos resultados dos dados, observou-se que, no que concerne às tecnologias inclusivas e assistivas, direcionadas ao público portador de deficiência intelectual e/ou mental, são poucas as tecnologias com registro de patente, em comparação a outras tecnologias existentes, tanto no mercado interno quanto no externo.

Também foi realizada uma busca na base de programas de computador do INPI usando-se a palavra-chave "assistiva". O resultado obtido foram apenas três registros de programa de computador, sendo que o registro BR 5120170001634 trata-se de um "sistema de robótica assistiva", o registro BR 5120170010366 trata-se de um "laboratório virtual de tecnologia assistiva" e o registro BR 5120180003966 trata-se de "casa assistiva".

A Tabela 1 detalha a quantidade de patentes depositadas nos mercados interno e externo, conforme os dados acima expostos.

Tabela 1 - Resultado das buscas Nacionais e Internacionais - INPI, CAPES, Orbit Questel

\begin{tabular}{|c|c|c|c|}
\hline \multirow[t]{2}{*}{ Palavras-chave } & \multicolumn{3}{|c|}{ NÚMERO DE DOCUMENTOS } \\
\hline & INPI & CAPES & ORBIT \\
\hline Defic* intelectual & 584 & 4.134 & 04 \\
\hline Deficiência and intelectual & 01 & 1.169 & 07 \\
\hline Deficiência intelectual & 238 & 2.614 & 02 \\
\hline Deficiência intelectual/ mental & 4.815 & 123 & 00 \\
\hline Portador de necessidade especial & 1.594 & 1.248 & 01 \\
\hline Tecnologia assistiva & 358 & 184 & 09 \\
\hline Assistiva & 03 & 219 & 00 \\
\hline Assistive technology & 00 & 17.660 & 272 \\
\hline Tecnologia assistiva educacional & 411 & 39 & 00 \\
\hline Assistiva educacional & 57 & 38 & 00 \\
\hline Educational assistive technology & 00 & 10.148 & 00 \\
\hline Tecnologia inclusiva & 362 & 700 & 00 \\
\hline Inclusive technology & 00 & 72.978 & 00 \\
\hline Tecnologia inclusiva educacional & 415 & 134 & 00 \\
\hline Inclusiva educacional & 53 & 478 & 00 \\
\hline Inclusive educational technology & 00 & 40.547 & 00 \\
\hline Inclusive educational & 00 & 62.012 & 01 \\
\hline
\end{tabular}

Fonte: Elaborada pelos autores deste artigo (2018)

Por seu turno, na investigação realizada na base de dados do Orbit Questel, com o uso das palavras-chave: "assistive technology", "tecnologia assistiva", "inclusive educational", foram encontrados 272 , nove e um processos de patenteamento, respectivamente. O exame das em- 
presas internacionais depositantes de patentes, segundo essa base de dados, segue a ordem especificada no Gráfico 1.

Gráfico 1 - Análise das empresas internacionais depositantes

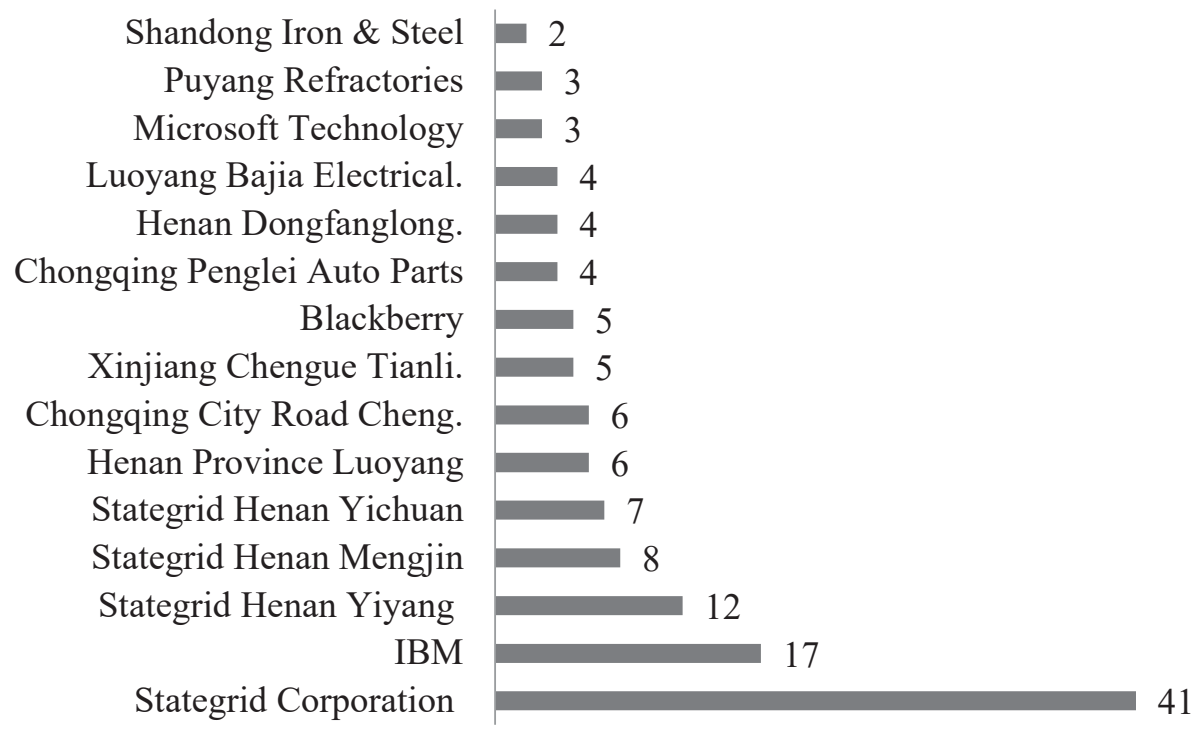

Fonte: Elaborado pelos autores deste artigo a partir da base de patentes do @O Orbit Questel (2018)

Com a utilização das palavras-chave escritas em inglês "assistive technology", observa-se que, em se tratando de tecnologia assistiva, a State Grid Corporation of China (SGCC) é a empresa detentora do maior número de depósitos de patentes entre PI e MU, seguida do grupo Luoyang Power Supply, com 17 reconhecimentos, e da IBM, com 12 patenteamentos na área da assistibilidade. Seguindo por esse caminho, percebe-se que os dados dos inventores mais atuantes estão expostos no Gráfico 2.

Gráfico 2 - Análise do ranking dos inventores mais influentes

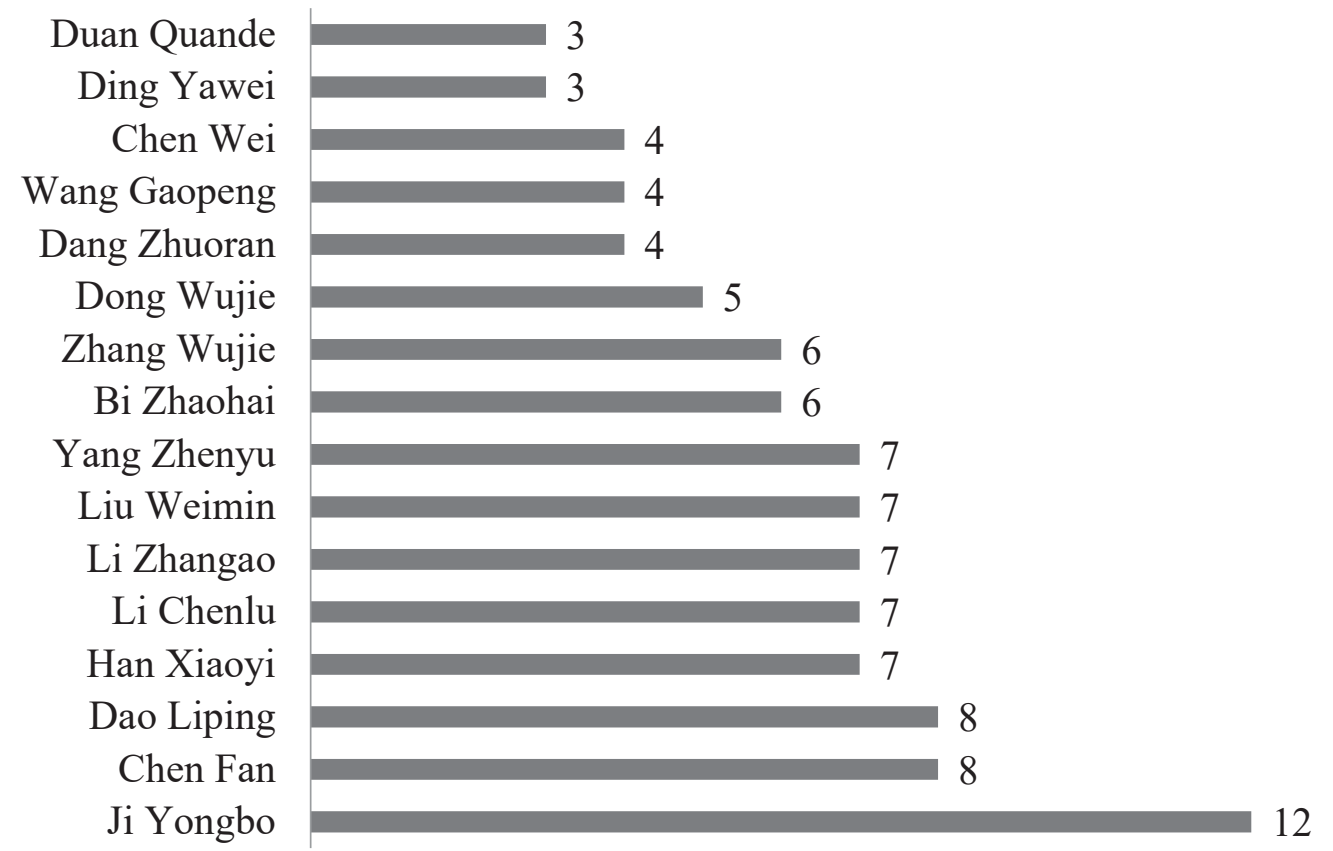

Fonte: Elaborado pelos autores deste artigo a partir da base de patentes do (COrbit Questel (2018) 
Com relação aos números investigados, não se observa a concentração de grandes empresas no desenvolvimento dessa tecnologia. A maior parte dos depósitos é realizada por inventores individuais e a presença de universidades também é tímida. Em continuidade, para facilitar a constatação da maior quantidade de processos de depósitos de patentes ao ano, o Gráfico 3 mostra a análise no período de 1999 a 2018.

Gráfico 3 - Análise das proteções no período 1999 a 2018

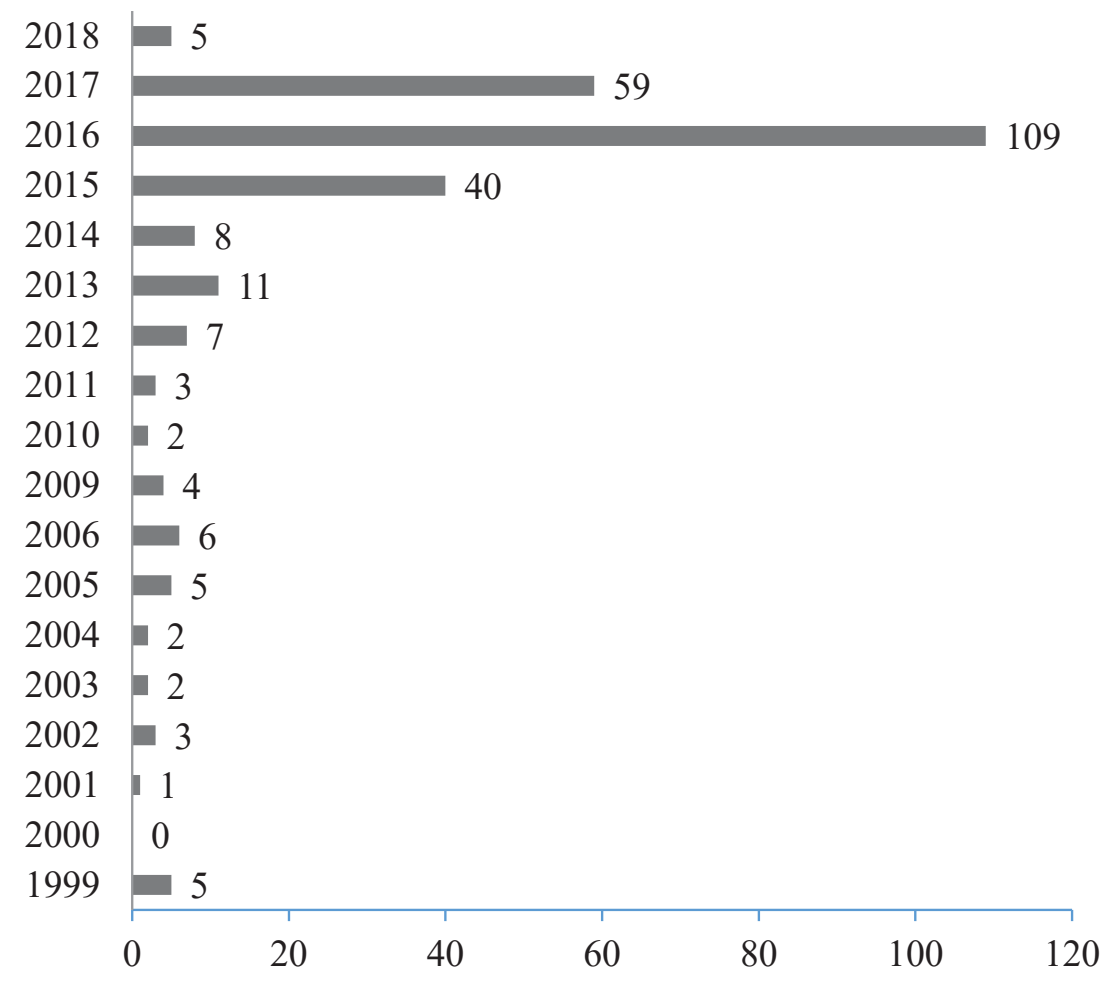

Fonte: Elaborado pelos autores deste artigo a partir da base de patentes do (c) Orbit Questel (2018)

Quando se observam as proteções por ano, período de 1999 a 2017, verifica-se no Gráfico 3 que há uma tendência de crescimento nas publicações, com oscilações no decorrer do período (anos de 1999 e 2005 (05), 2003, 2004 e 2010 (02), 2006 (06), 2015 (40)).

Os anos de 2016 e 2017 têm o maior volume de proteções, com 109 e 59 documentos, respectivamente, podendo tal fato ter sido motivado pela assinatura da Convenção sobre os Direitos das Pessoas com Deficiência da ONU pelos países signatários, no ano de 2005, e em decorrência disso, alguns países estarem instituindo leis próprias para estabelecer a inclusão da pessoa com deficiência. Desse modo, os estados-membros se comprometem em realizar e promover a pesquisa e o desenvolvimento de TAs destinadas a atender às necessidades específicas da PcD. 
Gráfico 4 - Domínio das tecnologias concedidas

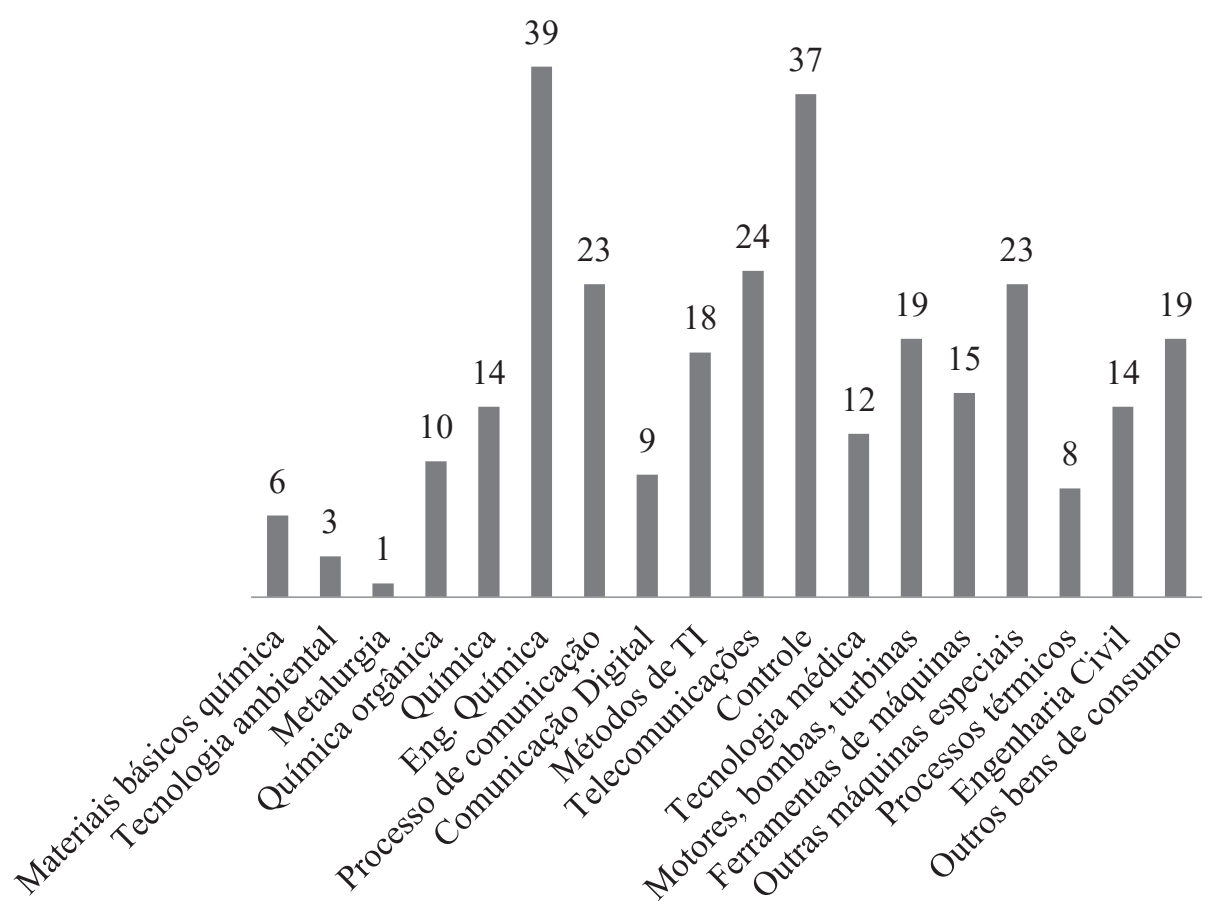

Fonte: Elaborado pelos autores deste artigo a partir da base de patentes do @O Orbit Questel (2018)

Quanto a isso, o domínio das TAs (Assistives Technologys) concentra-se em vários ramos da ciência, como: engenharia, medicina, química orgânica, comunicação digital, entre outros, com maior evidência nos números de patenteamento concedido em tecnologia de computadores (39) e tecnologia médica (37). Nenhuma tecnologia assistiva educacional para pessoas com deficiência intelectual e/ou mental foi encontrada.

Gráfico 5 - Análise dos países de publicação das patentes concedidas

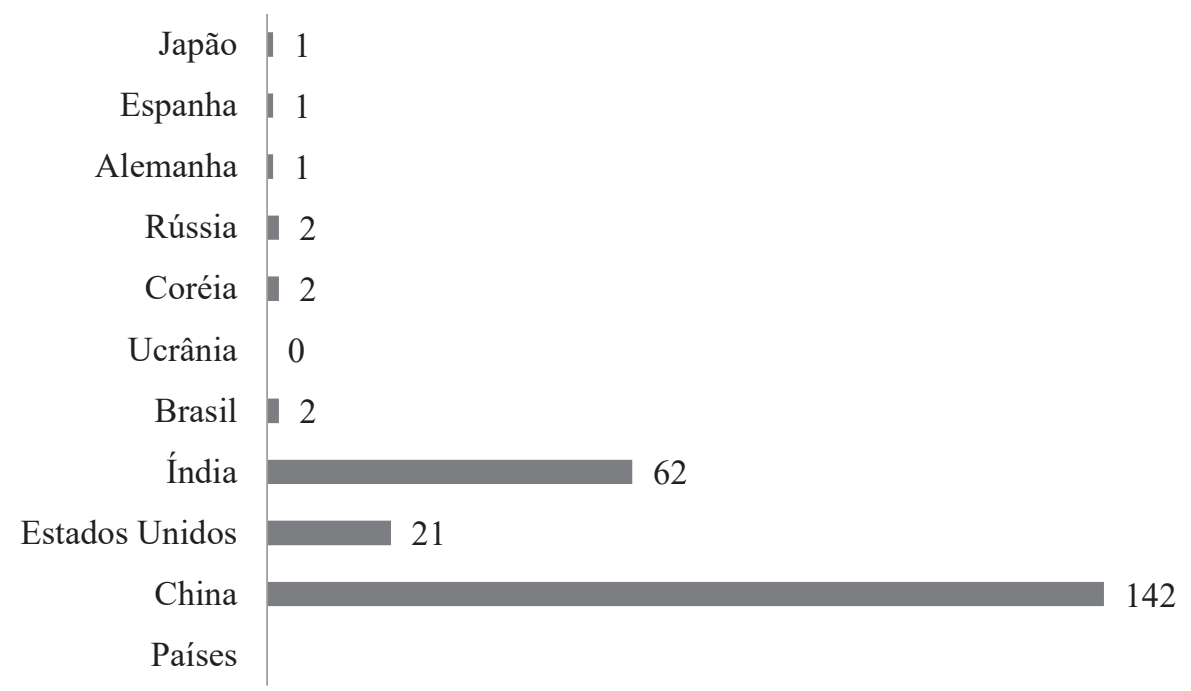

Fonte: Elaborado pelos autores deste artigo a partir da base de patentes do @OOrbit Questel (2018) 
A China foi o país que teve mais patentes concedidas e possui os principais responsáveis relacionados à tecnologia abordada, totalizando participação em 142 patentes analisadas. A Índia está em segundo lugar, com 62; os Estados Unidos estão em terceiro lugar com 21, e Brasil, Coreia e Rússia têm duas patentes. Segundo o site Xinhua Português (2019), a China trabalha arduamente para fortalecer ainda mais a proteção dos direitos de propriedade intelectual, com foco na proteção dos direitos e interesses legais de todos os tipos de segmentos de mercado. Como medida, a revisão da Lei de Patentes e a revisão do Regulamento de Implementação da Lei de Patentes estão em ritmo avançado.

Gráfico 6 - Condição das patentes concedidas

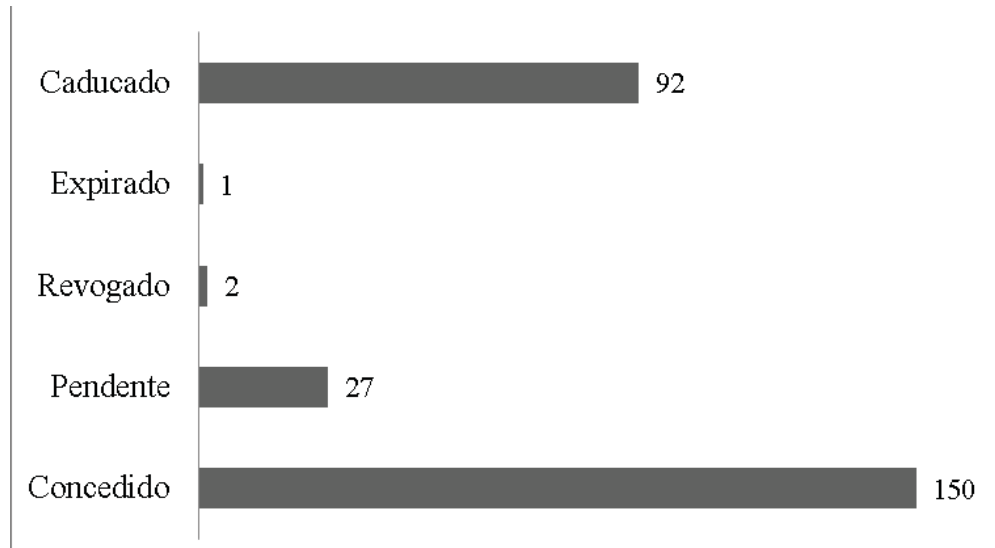

Fonte: Elaborado pelos autores deste artigo a partir da base de patentes do @O Orbit Questel (2018)

Quanto à condição das 272 patentes encontradas na base de dados do Orbit Questel, relativas à TA (Assistive Technology), elas estavam distribuídas da seguinte forma: 150 processos de patenteamento concedidos, ou seja, 55\%; 92 processos caducaram, perfazendo $34 \%$; 27 processos foram encontrados com pendência; dois estavam revogados; e apenas um havia expirado.

Na Figura 2, é possível visualizar uma organização a partir de uma Análise SWOT como instrumento para um planejamento estratégico, com a finalidade de recolher dados importantes que caracterizam os ambientes interno (forças e fraquezas) e externo (oportunidades e ameaças), antes de se investir na tecnologia.

Figura 2 - Análise sob a perspectiva da Matriz SWOT para a Tecnologia Assistiva educacional aplicada ao deficiente mental/intelectual
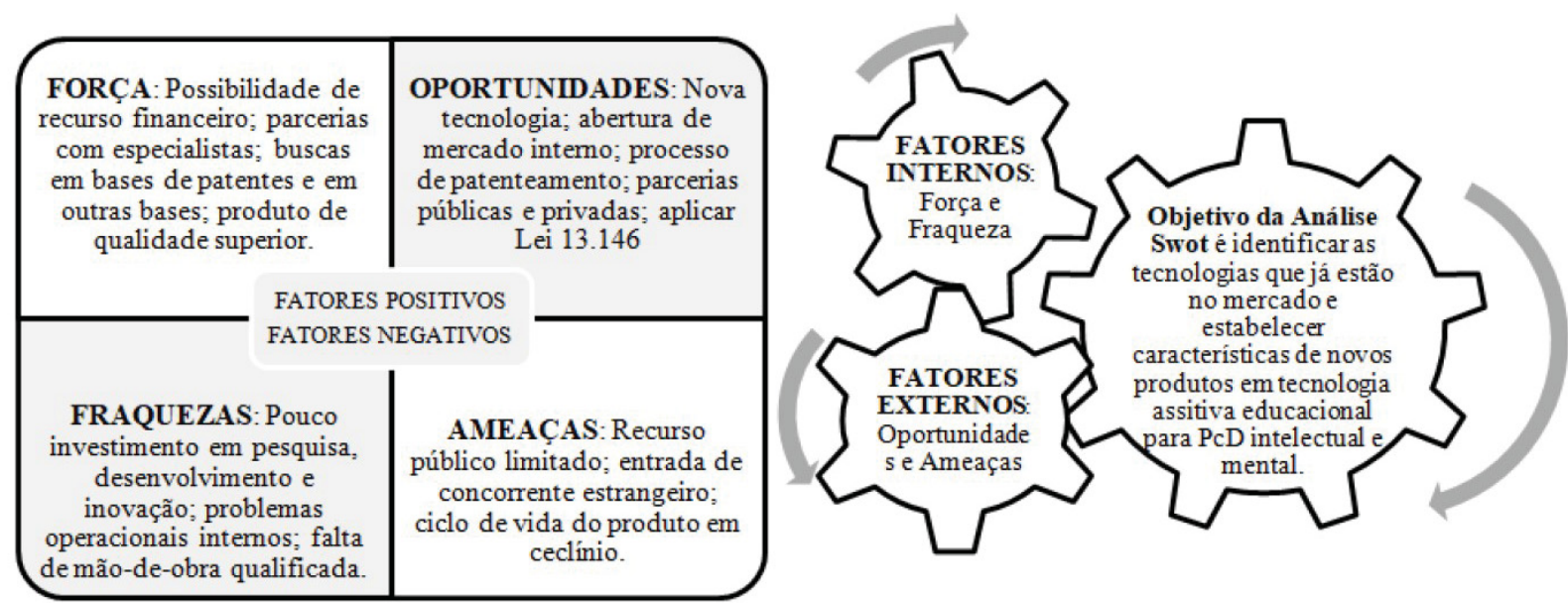

Fonte: Elaborada pelos autores deste artigo (2018) 
A Análise SWOT é uma ferramenta utilizada para fazer análise ambiental (interno e externo) e de cenário, com base na gestão e no planejamento de estratégia na empresa ou instituição, na presente situação, com a tecnologia assistiva educacional para PcD intelectual/ mental. Em vista de tal fato, essa foi uma das ferramentas utilizadas na fase Pós-Prospectiva desta pesquisa para tornar transparentes os fatores positivos e negativos, tanto externos quanto internos, encontrados durante a investigação.

Para se chegar ao resultado adequado desta análise, por meio da ferramenta SWOT, foi preciso dividir o ambiente onde se encontra a instituição em duas esferas: ambiente interno $e$ externo.

Ambiente interno (Forças e Fraquezas) - integração e padronização dos processos, eliminação de redundância e foco na atividade principal. O ambiente interno é formado pelo conjunto de recursos humanos, financeiros, físicos, parcerias com especialistas, entre outros, sobre os quais é possível exercer maior controle, pois são resultados de estratégias definidas pela direção.

Nesse ambiente é possível identificar os pontos fortes, correspondentes aos recursos e capacidades que, juntos, se transformam em uma vantagem competitiva frente aos seus concorrentes. E os pontos fracos, que são as deficiências que a instituição apresenta em comparação aos seus concorrentes atuais ou em potencial, como: pouco investimento em pesquisa, desenvolvimento e inovação; problemas operacionais internos; falta de mão de obra qualificada etc.

Ambiente externo (Oportunidades e Ameaças) - probidade e confiança nos dados, informação imediata de apoio à gestão e decisão estratégica e redução de erros. O ambiente externo é composto por fatores que existem fora dos limites da organização e, que de alguma forma, exercem influência sobre ela.

As "oportunidades" no ambiente externo são todas aquelas condições ambientais em que a instituição pode se beneficiar (propagar na mídia o evento, formar parcerias, nova tecnologia, registro de patente e cumprimento de legislação). Isso pode se dar pelo lucro ou por fatores que permitem atender a necessidades e desejos dos consumidores de forma vantajosa sobre a concorrência.

Por outro lado, como fator externo, encontra-se também a "ameaça", que pode ser determinada pela presença de recurso público limitado; entrada de concorrente no mercado; ciclo de vida do produto.

Assim, a Análise SWOT permite ao gestor identificar e transformar as possíveis fraquezas apresentadas no cenário tecnológico em oportunidades de identificar as tecnologias que já estão no mercado e estabelecer características de novos produtos em tecnológicos assistivos educacionais para $\mathrm{PcD}$ intelectual e/ou mental.

Finalmente, ao considerar que os fatores externos influenciam de forma homogênea nas instituições e empresas que atuam em um mesmo mercado, pode-se afirmar que somente aquelas que conseguirem melhor identificar as mudanças e tiverem agilidade para se adaptar é que conseguirão tirar melhor proveito das oportunidades sofrendo uma menor quantidade de danos e ameaças.

Ainda como estratégia de aplicação da gestão do conhecimento na fase Pós-Prospectiva, na Figura 3, é possível visualizar uma configuração da construção atual do Roadmap Tecnológico para a Tecnologia Assistiva Educacional para PcD intelectual/mental, em que são mostradas as partes envolvidas e o que será realizado em curto, médio e longo prazo. 
Figura 3 - Ponto Zero da Technology Roadmap referente à Tecnologia Assistiva aplicada ao deficiente intelectual/mental

\begin{tabular}{|c|c|c|c|}
\hline & & \multicolumn{2}{|c|}{ PRAZOS } \\
\hline & Curto & Médio & Longo \\
\hline \multicolumn{4}{|l|}{ ESTUDO DE PATENTES } \\
\hline \multicolumn{4}{|l|}{ Editor de texto } \\
\hline \multicolumn{4}{|l|}{ INPI } \\
\hline \multicolumn{4}{|l|}{ CAPES } \\
\hline \multicolumn{4}{|l|}{ Orbit } \\
\hline \multicolumn{4}{|l|}{ PRODUTOS } \\
\hline Software para computador & & $\mathbf{4}$ & \\
\hline \multicolumn{4}{|l|}{ Registro de software } \\
\hline \multicolumn{4}{|l|}{ Cartilha } \\
\hline \multicolumn{4}{|l|}{ Artigo científico } \\
\hline \multicolumn{4}{|l|}{ TECNOLOGIA } \\
\hline \multicolumn{4}{|l|}{ Word } \\
\hline \multicolumn{4}{|l|}{ Java ou PHP } \\
\hline \multicolumn{4}{|l|}{ Base de dados } \\
\hline \multicolumn{4}{|l|}{ Editor de texto } \\
\hline \multicolumn{4}{|l|}{ MERCADO } \\
\hline \multicolumn{4}{|l|}{ Escolas Públicas } \\
\hline \multicolumn{4}{|l|}{ Escolas Privadas } \\
\hline \multicolumn{4}{|l|}{ Unidades de Saúde } \\
\hline Prefeituras (serviços de assistência social) & & & \\
\hline
\end{tabular}

Fonte: Elaborada pelos autores deste artigo (2018)

Ainda na etapa Pós-Prospectiva da presente pesquisa, foi elaborado, para melhor logística e esclarecimento da TA, o roadmap tecnológico, com o propósito de projetar os prazos (curto, médio e longo), no cenário tecnológico, e melhorar a administração do estudo de patentes, produtos, tecnologia e mercado nos ambientes nacional e internacional.

Constituído do ponto zero, o que concerne ao estudo de patentes, relacionado à TA para educação de $\mathrm{PcD}$ intelectual/ mental requereu: editor de texto, consultas às bases de dados nacionais e internacionais como INPI, CAPES, Orbit, estabelecido em curto prazo para a demanda.

Em médio prazo, foram organizados os produtos frutos da pesquisa, constituídos de um software, a ser entregue em médio prazo, e registro em longo prazo; cartilha para médio prazo e artigo científico em longo prazo. Para o desenvolvimento da tecnologia, foi esboçada a necessidade de uso da Web, Java ou PHP, base de dados, editor de texto, no decorrer de todos os prazos, ou seja, curto, médio e longo.

Por fim, no que concerne ao mercado, os envolvidos são: escolas públicas, escolas privadas, unidades de saúde, Prefeituras (Serviços de Assistência Social), estes em longo prazo.

A técnica de technology roadmapping é muito efetiva na conexão do planejamento e estratégia de negócios que envolvem tecnologias, onde esse planejamento depende, na maior parte 
das vezes, da análise qualitativa de técnicos na área especifica. Contudo, a análise e a avaliação unicamente qualitativa do Roadmap pode não revelar todas as informações necessárias quanto às tendências mercadológica, tecnológica e estratégica das potenciais empresas e dos possíveis investidores.

\section{Considerações Finais}

Diante dos números nacionais quanto às $\mathrm{PcDs}$, ressaltando os deficientes intelectuais/ mentais, que compreendem cerca de $24 \%$ da população brasileira, a inserção das tecnologias assistivas na educação escolar poderá proporcionar uma melhora na qualidade de vida e promover a inclusão social dessa parcela da população, bem como, a longo prazo, atenuar possíveis progressos da deficiência.

Verificou-se que a elaboração de uma boa estratégia para a realização da busca de documentos de patentes pode auxiliar bastante na compilação dos dados, assim como as técnicas de prospecção para melhor compreender e administrar o ambiente (externo e interno) e o estudo do cenário para boa gestão da instituição com o investimento na pesquisa da tecnologia assistiva.

A recente introdução dos termos "Tecnologias Assistivas" não apresenta ainda bons resultados para busca, muito menos as buscas de TA educacionais para PcD intelectual/mental, exceto para a área de medicamentos, informática, biotecnologia e química.

Não há concentração de grandes empresas e universidades no desenvolvimento dessa tecnologia, e a maioria dos depósitos é realizada por inventores individuais, evidenciando que pode estar ocorrendo uma não vinculação dos inventores com as empresas, ou essas tecnologias são desenvolvidas e transferidas ou não protegidas (SANTOS et al., 2015).

Percebe-se que há necessidade de estímulos de iniciativas públicas e privadas para o aumento das PD\&I voltadas para as tecnologias assistivas educacionais ao público com deficiência intelectual e mental, por se tratar de uma questão de qualidade de vida, cidadania e, principalmente, de meios para facilitar o cumprimento da Legislação Nacional direcionada às Pessoas Portadoras de Deficiência.

\section{Referências}

$\mathrm{BERSCH}, \mathrm{R}$. Introdução à tecnologia assistiva. CEDI - Centro Especializado em Desenvolvimento Infantil. Assistiva, Tecnologia e Educação. Porto Alegre, RS, 2017. Disponível em: http://www.assistiva.com.br/Introducao_Tecnologia_Assistiva.pdf. Acesso em: 17 dez. 2018.

BORSCHIVER, S.; LEMOS, A. Technology Roadmap: Planejamento Estratégico para alinhar Mercado - Produto - Tecnologia. Rio de Janeiro: Ed. Interciência, 2016.

BRASIL. Lei n. 13.146, de 6 de julho de 2015. Lei Brasileira de Inclusão da Pessoa com Deficiência (Estatuto da Pessoa com Deficiência), Brasília, DF, jul. 2015. Disponível em: http://www. planalto.gov.br/CCIVIL_03/_Ato2015-2018/2015/Lei/L13146.htm. Acesso em: 13 dez. 2018.

CARDOSO, F.; BOMTEMPO, J. V.; BORSCHIVER, S. Elaboração de Roadmap tecnológico para a produção de biogás a partir da vinhaça. Cadernos de Prospecção, Salvador, v. 10, n.3 p. 495-509, jul.-set. 2017. D.O.I.: http://dx.doi.org./10.9771/cp.v10i3.22929. 
CGEE - CENTRO DE GESTÃO E ESTUDOS ESTRATÉGICOS. 2014. Disponível em: http:/ www.cgee.org.br/prospeccao/index.php?operacao=Exibir\&serv=textos/topicos/texto_exib\&tt o_ $\mathrm{id}=4 \&$ tex_id=1. Acesso em: $16 \mathrm{dez} .2018$.

COELHO, M. G. Prospecção Tecnológica: Metodologias e Experiências Nacionais e Internacionais. Rio de Janeiro: [s.n.], 2003. Disponível em: http://www.cgee.org.br/atividades/redirKori/462. Acesso em: 17 dez. 2018.

IBGE - INSTITUTO BRASILEIRO DE GEOGRAFIA E ESTATÍSTICA. 2019. Disponível em: https://sidra.ibge.gov.br/tabela/3426\#/n1/all/n3/26,29/v/allxp/p/all/c134/all/c2/all/c86/0/d/v93\%20 0/l/v,p+t+c2,c86+c134/resultado. Acesso em: 2 dez. 2018.

INPI - INSTITUTO NACIONAL DA PROPRIEDADE INDUSTRIAL. [2018a]. Disponível em: http:// www.inpi.gov.br/menu-servicos/patente/guia-basico-de-patentes. Acesso em: 17 dez. 2018.

INPI - INSTITUTO NACIONAL DA PROPRIEDADE INDUSTRIAL. [2018b]. Disponível em: https:// gru.inpi.gov.br/pePI/servlet/PatenteServletController. Acesso em: 12 dez. 2018.

KINTSCHNER, F. E.; FERREIRA, D. H. L. Tecnologias da informação e comunicação a serviço de pessoas com deficiência. [S.l.]: Brazilian Technology Symposium, 2018. v. 1.

ORBIT. Questel Orbit. [2018]. Disponível em: https://www63.orbit.

$\mathrm{com} /$ ?locale $=$ en\&ticket $=$ ab9b2192-1818-418a-8e22-2b2c74ae8907\&embedded $=$ false \#PatentSear chHistoryPage. Acesso em: 12 dez. 2018.

SANTOS, J. C. et al. Estudo prospectivo de tecnologias assistivas educacionais para pessoas com deficiência visual. Cadernos de Prospecção, Salvador, v. 8, n. 1, p. 35-45, jan.-mar. 2015. DOI: 10.9771/S. CPROSP. 2015.008.005.

TATUM, C. T. S. et al. Potencialidades e aplicações do jamelão, Syzygium Cumini. In: 9TH INTERNATIONAL SYMPOSIUM ON TECHNOLOGICAL INNOVATION (ISTI ) SIMTEC, Aracajú, SE, v. 8, n. 1, p. 1-3, set. 2018. Anais [...], Aracajú, SE, v. 8, n. 1, p. 1-3, set. 2018. DOI 10.71981S2318-3403201600030001.

XINHUA PORTUGUÊS. [2019]. Disponível em: http://portuguese.xinhuanet.com/201907/18/c_138236581.htm. Acesso em: 22 nov. 2019.

\section{Sobre as Autoras}

\section{Júlia Maciela Oliveira de Tassis Frasson Cardozo}

E-mail: juliamaciela@hotmail.com

Especialista em Direito Público e Direito Privado pela Faculdade de Tecnologia e Ciências de Vitória da Conquista Bahia em 2019.

Endereço profissional: Universidade Federal do Vale do São Francisco, Campus Juazeiro, Avenida Antônio Carlos Magalhães, n. 510, Santo Antônio, Juazeiro, BA. CEP: 48902-300.

\section{Cristiane Xavier Galhardo}

E-mail: Cristiane.galhardo@univasf.edu.br

Pós-Doutorado em Química Analítica pelo Centro de Energia Nuclear na Agricultura em 2003. Doutora em Ciências (Química Analítica) pela Universidade de São Paulo em 2001.

Endereço profissional: Federal do Vale do São Francisco, Campus Juazeiro, Avenida Antônio Carlos Magalhães, n. 510, Santo Antônio, Juazeiro, BA. CEP: 48902-300. 


\section{Vivianni Marques Leite dos Santos}

E-mail: vivianni.santos@gmail.com

Doutorado em Química pela Universidade Federal de Pernambuco em 2004.

Endereço profissional: Universidade Federal do Vale do São Francisco, Campus Juazeiro, Avenida Antônio Carlos Magalhães, n. 510, Santo Antônio, Juazeiro, BA. CEP: 48902-300. 